\title{
Contas Nacionais
}

\section{National Accounts}

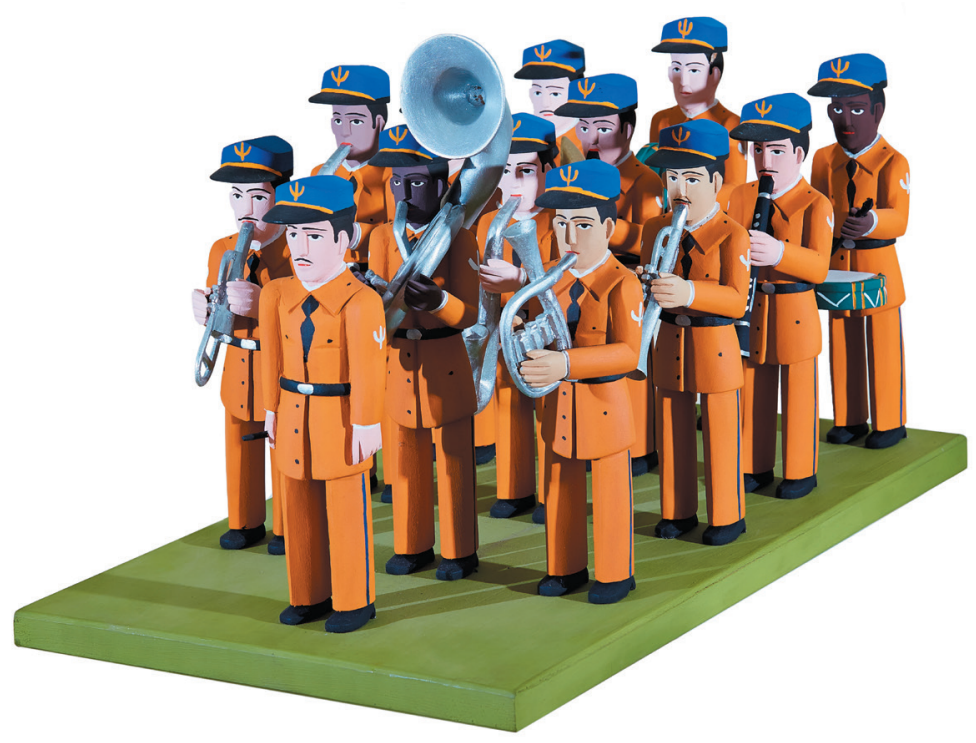

Banda/ Marching band, 2014

Geicifran Francisco de Assis Azevedo 



\section{Contas Nacionais}

Em 2015, a economia brasileira vivenciou um agravamento da conjuntura recessiva iniciada no ano anterior, registrando uma variação anual de $-3,8 \%$ no Produto Interno Bruto (PIB), o que veio a se constituir no pior resultado da produção de bens e serviços na era do Real. Com isso, o PIB a preços de mercado totalizou $\mathrm{R} \$ 5.904 .331$ milhões (Tabela 10.1) $\mathrm{A}$ deterioração da atividade produtiva tornou-se evidente ao longo do ano, porquanto a comparação do PIB em cada trimestre com o período correspondente no ano anterior mostrou taxas crescentemente negativas, evoluindo de uma queda de $-2,0 \%$ no primeiro trimestre para uma variação de $-5,9 \%$ no último trimestre e completando, assim, sete períodos ininterruptos com taxas trimestrais negativas (Tabela 10.4). Esta piora nas condições de produção impactou no mercado de trabalho - o nível geral de emprego diminuiu e a taxa de desemprego sofreu rápida elevação -, bem como se fez acompanhar por um aumento na taxa de inflação que fechou o ano acima do limite superior da meta. Neste contexto, o PIB per capita a preços correntes oscilou de R\$28.046 em 2014 para R\$ 28.846 em 2015 (Tabela 10.1), numa variação de apenas $2,8 \%$, bem

\section{National Accounts}

In 2015, the Brazilian economy experienced a deterioration of the recessive conditions observed since the previous year. The Gross Domestic Product (GDP) recorded a negative change of $3.8 \%$ in real terms, which was the worst outcome regarding the production of goods and services throughout the period of Real. As a consequence, GDP reached $\mathrm{R} \$$ $5,904,331$ million. The slump in the productive activity became evident during the year, for quarterly rate changes showed increasing negative results from $-2.0 \%$ in the 1 st quarter down to $-5.9 \%$ in the 4 th quarter. Thus, the Brazilian economy completed seven uninterrupted periods of negative quarterly rate changes (Table 10.4). This downward trend in the conditions of production affected the labor market - the general level of employment decreased and the rate of unemployment showed a rapid increase - as well as went along with an increase in the rate of inflation to a level beyond the upper limit of the target. Under these circumstances, the GDP per capita fluctuated from $\mathrm{R} \$ 28,046$ in 2014 to $R \$$ 28,846 in 2015 at current prices (Table 10.1 ), changing by only $2.8 \%$, a rate well 
inferior ao crescimento dos preços em geral.

O recuo na atividade produtiva tem origem em múltiplos fatores, incluindo aqueles presentes há mais tempo como a continuidade da política monetária contracionista. Em 2015, delineou-se também uma política fiscal de mesmo tipo, baseada em contenção nos gastos e reversão em benefícios fiscais, que afetou diretamente a demanda agregada. Abandonou-se, assim, a combinação de políticas observada nos anos imediatamente anteriores, quando a condução da política fiscal procurou reduzir os efeitos contracionistas da política monetária e da instabilidade no cenário externo. Além disso, do lado da oferta, o efeito de uma severa seca sobre as condições de geração de energia mostrou-se mais intenso do que fora previsto, ao passo que, no setor externo, a economia brasileira veio a ser negativamente impactada pela redução nos preços das commodities.

O conjunto de condições desfavoráveis à atividade produtiva pesou sobre o já precário estado de confiança dos setores institucionais, atingindo as decisões de consumo e investimento. A despesa de consumo das famílias cresceu apenas 5,5\% em valores correntes, chegando a R\$3.741.855 milhões em 2015 (Tabela 10.3). Dado que os preços ao consumidor aumentaram em ritmo bastante superior, conclui-se que houve uma redução real no consumo familiar em conformidade com a piora generalizada no mercado de trabalho e na confiança do consumidor. $\mathrm{O}$ aumento de um ponto percentual sobre o PIB resultou meramente de uma menor diminuição below the increase in the general level of prices.

This decline in the production activity stemmed from multiple factors, including those in progress for a longer period such as the continuity of a restrictive monetary policy. In 2015, a fiscal policy of similar nature was adopted through measures for both slowing expenditures down and reversing tax benefits, which exerted a direct impact on the aggregate demand. By so proceeding, the government has abandoned the combination of policies carried on in previous years, according to which fiscal policy was set to minimize the contraction effects of both the monetary policy and the instability of the external sector. Moreover, on the supply side of the economy, the effects of a severe drought over the conditions to generate power proved stronger than they had been speculated, whereas, as for the external sector, the Brazilian economy was negatively affected by a drop in the price of commodities.

All these objective conditions detrimental to the production activity put more pressure over an already precarious degree of confidence of the institutional sectors, having an adverse effect on the decisions of consumption and investment. Thus, household consumption grew by only $5.5 \%$ at current prices, reaching $R \$ 3,741,855$ million in 2015 (Table 10.3). Since the increase of consumer prices was greater, this brought about a real contraction in household consumption, which conforms to the overall worsening of both the labor market and the consumer confidence. An increase of household consumption by one percentage point over the GDP was a result of a lower 
relativa do consumo vis-à-vis o produto, a preços constantes.

As consequências do quadro econômico adverso evidenciaram-se de modo ainda mais agudo no desempenho da formação bruta de capital, que declinou de R\$ 1.186.631 milhões em 2014 para R\$ 1.045.771 milhões em 2015, em valores correntes (Tabela 10.3). Isto levou a taxa de investimento para a casa dos $18,2 \%$ do PIB, fixando-a num patamar entre dois e três pontos percentuais abaixo do que se observara em anos recentes (Tabela 10.5). A par das consequências negativas sob a ótica do dispêndio, a redução no volume e na taxa de investimento também ocasiona uma piora nas condições de crescimento da capacidade produtiva e dos níveis gerais de produtividade da economia brasileira.

\section{A dinâmica recessiva da economia} conduziu ao aprofundamento do processo de desindustrialização do país, evidenciado por uma menor participação da indústria na formação do produto. As taxas trimestrais correspondentes ao produto da indústria mostraram-se sistemática e crescentemente negativas, passando de $-4,4 \%$ no primeiro trimestre até atingir $-8,0 \%$ no quarto trimestre (Tabela 10.4). Com isso, a participação do setor industrial no produto recuou 1,3 pontos percentuais e fechou o ano em nível correspondente a $22,7 \%$ do valor adicionado a preços básicos. Já o setor de serviços aumentou sua participação para $72,1 \%$, pois, dentre outros motivos, a redução no seu produto foi menos pronunciada do que a do produto em geral. O setor da agropecuária manteve estável sua participação em torno a 5,2\% do valor adicionado a preços correntes (Tabela 10.2). negative change vis-à-vis the GDP at constant prices.

The consequences of this adverse economic context were sharply felt in an appalling performance of gross capital formation, which shrank from $\mathrm{R} \$ 1,186,631$ million in 2014 to $\mathrm{R} \$$ $1,045,771$ million in 2015 at current prices (Table 10.3). This lessened the investment rate to $18.2 \%$ of the GDP, a level between two and three percentage points below the previous years (Table 10.5). In addition to its negative consequences over aggregate demand, the reduction in investment also aggravated the conditions for both the expansion of productive capacity and the increase in the general productivity levels of the Brazilian economy.

The recessive economic performance has deepened the process of deindustrialization of the country, which may be evidenced in an even smaller proportion of the manufacturing product over the general economy added value. Quarterly rates regarding manufacturing, mining and quarrying showed increasingly negative changes, oscillating from $-4.4 \%$ in the 1 st quarter down to $-8.0 \%$ in the 4 th quarter (Table 10.4). As a consequence, the proportion of manufacturing over the product curtailed by 1.3 percentage points, closing the year in a level equal to $22.7 \%$ of added value at basic prices. As for the services sector, it accounted for $72.1 \%$ of added value in 2015, rising from a lower figure in the previous year in spite of displaying negative changes in every quarterly rates. Agriculture, forestry and fishing kept unchanged its proportion in $5.2 \%$ of added value at current prices (Table 10.2). 
De modo diverso ao que se verificou no front interno, houve uma melhora no desempenho de bens e serviços no setor externo. Conforme exposto na Tabela 10.3 , enquanto as importações oscilaram $6,8 \%$ em valores correntes, atingindo $\mathrm{R} \$$ 845.779 milhões em 2015, as exportações cresceram do modo mais vigoroso $(+21,0 \%)$ e chegaram $\mathrm{R} \$ 770.084$ milhões, reduzindo o saldo negativo das exportações líquidas para menos de metade do que se observara em 2014. O grau de abertura da economia, medido pelo fluxo total de comércio, passou de $25,1 \%$ para $27,4 \%$ (Tabela 10.5). Estes resultados refletem, de um lado, o desaquecimento observado na absorção interna, em que a soma do consumo final e do investimento aumentou tão somente $2,3 \%$ em valores correntes (Tabela 10.1), freando o crescimento mais rápido das importações, e, de outro lado, as consequências do movimento de depreciação real da moeda doméstica que se sustentou durante o ano de 2015.

A melhora no saldo das operações com bens e serviços levou a uma expressiva redução na necessidade líquida de financiamento da economia brasileira, que passou de $\mathrm{R} \$ 263.600$ milhões em 2014 para R\$ 194.708 milhões em 2015, a preços correntes (Tabela 10.1). Conforme se verifica no comportamento dos agregados de renda da Tabela 10.1, a economia nacional manteve seu padrão estrutural em que a Renda Nacional Bruta é menor do que o PIB em face do saldo negativo na renda primária, mormente devido a pagamentos líquidos de juros e lucros. Esta renda líquida enviada ao exterior, no entanto, não se ampliou de modo significativo entre 2014 e 2015, de modo que a dinâmica das exportações líquidas foi a principal responsável pela
Transactions with goods and services have showed a better performance in the external sector vis-à-vis the domestic front. According to Table 10.3, whereas imports increased by $6.8 \%$ at current prices, reaching $\mathrm{R} \$ 845,779$ million in 2015, exports enjoyed a greater rise $(21.0 \%)$ to $\mathrm{R} \$ 770,084$ million, cutting down the negative balance of net exports by more than $50 \%$ in contrast to the 2014 value. The degree of openness of the economy, as measured by to total flow of trade, grew larger from $25.1 \%$ to $27.4 \%$ (Table 10.5 ). These outcomes reflected, on the one hand, the slowdown in the domestic demand, according to which the sum of final consumption and investment increased by no more than $2.3 \%$ at current prices (Table 10.1), curbing a more vigorous rise in imports, and, on the other hand, the consequences of a process of real depreciation of the domestic currency that lasted for the whole year of 2015 .

The improvement in the net balance of goods and services brought about a sizeable reduction in net borrowing, which changed from $R \$ 263,600$ million in 2014 to $R \$ 194,708$ million in 2015 at current prices (Table 10.1). By looking at the aggregates of income in Table 10.1 , one can observe that the Brazilian economy kept unchanged a structural pattern according to which the Gross National Income is lower than GDP due to a negative balance in primary income, especially concerning net payments of interest and transfers of profits. However, the increase in the net income sent to the rest of the world cannot be considered expressive in 2015 , so that it was mainly the performance of the trade balance that 
contração da necessidade líquida de financiamento em mais de $25 \%$ do valor registrado no ano anterior.

A redução no investimento em R\$ 140.860 milhões fez-se acompanhar não apenas pela menor necessidade líquida de financiamento, como por uma diminuição na poupança bruta, que passou de R\$ 922.476 milhões em 2014 para R\$ 859.535 milhões em 2015 em valores correntes (Tabela 10.1). caused a decrease in net borrowing by more than $25 \%$ in comparison to the previous year.

The reduction in investment by $\mathrm{R} \$ 140,860$ million was followed not only by a lower net borrowing, but also by a decrease in gross saving, which oscillated from $\mathrm{R} \$ 922.476$ million in 2014 to $\mathrm{R} \$ 859.535$ million in 2015 at current prices (Table 10.1).

\section{Carlos Henrique Horn}

Mestre em economia pela Universidade Federal do Rio Grande do Sul - UFRGS Doutor em relações de trabalho pela London School of Economics and Political Science - LSE, da Universidade de Londres Professor Associado da Faculdade de Ciências Econômicas da Universidade Federal do Rio Grande do Sul - UFRGS
M.Sc. Economics, Federal University of Rio Grande do Sul - UFRGS

Ph.D. Industrial Relations, London School of Economics and Political Science - LSE, University of London

Associate Professor, School of Economic Sciences, Federal University of Rio Grande do Sul - UFRGS 
Tabela 10.1 - Principais agregados macroeconômicos - 2013-2015

Table 10.1 - Main macroeconomic aggregates - 2013-2015

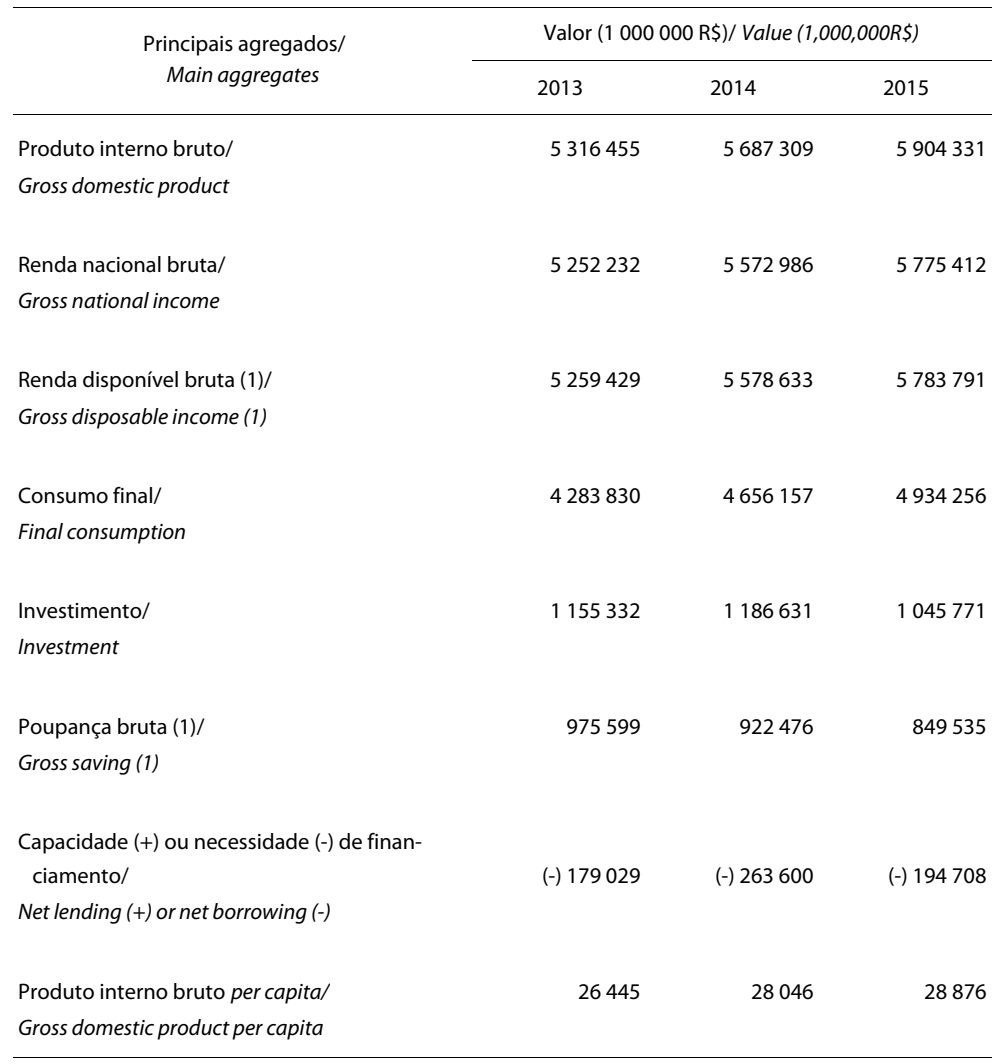

Fontes/Sources: 1. Sistema de contas nacionais: Brasil: 2010-2013. Rio de Janeiro: IBGE, 2015. (Contas nacionais, n. 46). Disponível em/Available from: <http://www.ibge.gov.br/home/estatistica/economia/ contasnacionais/2013/default.shtm>. Acesso em: mar. 2016/Cited: Mar. 2016. 2. Indicadores IBGE: contas nacionais trimestrais: indicadores de volume e valores correntes out./dez. 2015. Rio de Janeiro: IBGE, 2016. Disponível em/Available from: <http://www.ibge.gov.br/home/estatistica/indicadores/ pib/defaultcnt.shtm>. Acesso em: mar. 2016/Cited: Mar. 2016.

Nota: Os dados de 2014 e 2015 são preliminares baseados em Contas Nacionais Trimestrais./ Note: Preliminary data for 2014 and 2015 based on the Quarterly National Accounts.

(1) Inclui as transferências de capital por impossibilidade de identificá-las, até o momento./ (1) Includes capital transfers due to the impossibility of identifying them up to now. 
Tabela 10.2 - Participação percentual dos impostos e do valor adicionado, a preços básicos no Produto Interno Bruto - PIB, e dos setores de atividade, no valor adicionado a preços básicos - 2013-2015

Table 10.2 - Percentage participation of the taxes and of the value added, at basic prices in the Gross Domestic Product - GDP, and of the sectores of activity, in value added at basic prices - 2013-2015

\begin{tabular}{|c|c|c|c|}
\hline \multirow{2}{*}{$\begin{array}{l}\text { Especificação/ } \\
\text { Item }\end{array}$} & \multicolumn{3}{|c|}{ Participação percentual (\%)/ Percent participation (\%) } \\
\hline & 2013 & 2014 & 2015 \\
\hline $\begin{array}{l}\text { Produto interno bruto/ } \\
\text { Gross domestic product }\end{array}$ & 100,0 & 100,0 & 100,0 \\
\hline $\begin{array}{l}\text { Impostos/ } \\
\text { Taxes }\end{array}$ & 14,6 & 14,2 & 14,4 \\
\hline $\begin{array}{l}\text { Valor adicionado a preços básicos / } \\
\text { Value added at basic price }\end{array}$ & 85,4 & 85,8 & 85,6 \\
\hline $\begin{array}{l}\text { Valor adicionado a preços básicos / } \\
\text { Value added at basic prices }\end{array}$ & 100,0 & 100,0 & 100,0 \\
\hline $\begin{array}{l}\text { Agropecuária/ } \\
\text { Agriculture, forestry and fishing }\end{array}$ & 5,3 & 5,2 & 5,2 \\
\hline $\begin{array}{l}\text { Indústria (1)/ } \\
\text { Manufacturing, mining and quarrying }\end{array}$ & 24,9 & 24,0 & 22,7 \\
\hline $\begin{array}{l}\text { Serviços/ } \\
\text { Services }\end{array}$ & 69,8 & 70,8 & 72,1 \\
\hline
\end{tabular}

Fontes/Sources: 1. Sistema de contas nacionais: Brasil: 2010-2013. Rio de Janeiro: IBGE, 2015. (Contas nacionais, n. 46). Disponível em/Available from: <http://www.ibge.gov.br/home/estatistica/economia/ contasnacionais/2013/default.shtm>. Acesso em: mar. 2016/Cited: Mar. 2016. 2. Indicadores IBGE: contas nacionais trimestrais: indicadores de volume e valores correntes out./dez. 2015. Rio de Janeiro: IBGE, 2016. Disponível em/Available from: <http://www.ibge.gov.br/home/estatistica/indicadores/ pib/defaultcnt.shtm>. Acesso em: mar. 2016/Cited:Mar. 2016.

Nota: Os dados de 2014 e 2015 são preliminares baseados em Contas Nacionais Trimestrais./ Note: Preliminary data for 2014 and 2015 based on the Quarterly National Accounts.

(1) Inclusive eletricidade, gás, água e construção./ (1) Includes electricity, gas, water and construction. 


\section{Tabela 10.3 - Composição do Produto Interno Bruto - PIB, sob a ótica da despesa - 2013-2015 \\ Table 10.3 - Composition of Gross Domestic Product - GDP, considering expenditures - 2013-2015}

\begin{tabular}{|c|c|c|c|c|c|c|}
\hline \multirow{2}{*}{$\begin{array}{l}\text { Composição/ } \\
\text { Composition }\end{array}$} & \multicolumn{3}{|c|}{$\begin{array}{l}\text { Valor (1 } 000000 \mathrm{R} \$) / \\
\text { Value }(1,000,000 \mathrm{R} \$)\end{array}$} & \multicolumn{3}{|c|}{$\begin{array}{l}\text { Percentual do PIB (\%)/ } \\
\text { Percent of GDP (\%) }\end{array}$} \\
\hline & 2013 & 2014 & 2015 & 2013 & 2014 & 2015 \\
\hline $\begin{array}{l}\text { Produto interno bruto/ } \\
\text { Gross domestic product }\end{array}$ & 5316455 & 5687309 & 5904331 & 100,00 & 100,00 & 100,00 \\
\hline $\begin{array}{l}\text { Consumo final/ } \\
\text { Final consumption }\end{array}$ & 4283830 & 4656157 & 4934256 & 80,58 & 81,87 & 83,57 \\
\hline $\begin{array}{l}\text { Despesa de consumo das } \\
\text { famílias (1)/ }\end{array}$ & 3276050 & 3547428 & 3741855 & 61,62 & 62,37 & 63,37 \\
\hline $\begin{array}{l}\text { Final consumption expenditure of } \\
\text { households (1) }\end{array}$ & & & & & & \\
\hline $\begin{array}{l}\text { Despesa de consumo da administ } \\
\text { ção pública/ }\end{array}$ & 1007780 & 1108729 & 1192401 & 18,96 & 19,49 & 20,20 \\
\hline $\begin{array}{l}\text { General Government final consump } \\
\text { expenditure }\end{array}$ & & & & & & \\
\hline $\begin{array}{l}\text { Formação bruta de capital/ } \\
\text { Gross capital formation }\end{array}$ & 1155332 & 1186631 & 1045771 & 21,73 & 20,86 & 17,71 \\
\hline $\begin{array}{l}\text { Exportação de bens e serviços/ } \\
\text { Exports of goods and services }\end{array}$ & 620077 & 636230 & 770084 & 11,66 & 11,19 & 13,04 \\
\hline $\begin{array}{l}\text { Importação de bens e serviços (-)/ } \\
\text { Imports of goods and services (-) }\end{array}$ & (-) 742784 & (-) 791709 & (-) 845779 & (-) 13,97 & (-) 13,92 & (-) 14,32 \\
\hline
\end{tabular}

Fontes/Sources: 1. Sistema de contas nacionais: Brasil: 2010-2013. Rio de Janeiro: IBGE, 2015. (Contas nacionais, n. 46). Disponível em/Available from: <http://www.ibge.gov.br/home/estatistica/economia/ contasnacionais/2013/default.shtm>. Acesso em: mar. 2016/Cited: Mar. 2016. 2. Indicadores IBGE: contas nacionais trimestrais: indicadores de volume e valores correntes out./dez. 2015. Rio de Janeiro: IBGE, 2016. Disponível em/Available from:<http://www.ibge.gov.br/home/estatistica/indicadores/pib/ defaultcnt.shtm>. Acesso em: mar. 2016/Cited: Mar. 2016.

Nota: Os dados de 2014 e 2015 são preliminares baseados em Contas Nacionais Trimestrais./ Note: Preliminary data for 2014 and 2015 based on the Quarterly National Accounts.

(1) Os dados de consumo das famílias incluem o consumo das famílias + despesa de consumo das instituições sem fins de lucro a serviço das famílias./ (1) Data for household consumption include the household final consumption expenditure + consumption non profit institutions serving households final consumption expenditure. 
Tabela 10.4 - Variação da taxa trimestral do Produto Interno Bruto - PIB, por setor de atividade - 2014-2015

Table 10.4 - Quarterly rate change of the Gross Domestic Product - GDP,

by sector of activity - 2014-2015

\begin{tabular}{|c|c|c|c|c|c|c|c|c|}
\hline \multirow[b]{3}{*}{$\begin{array}{l}\text { Setor de atividade/ } \\
\text { Sector of activity }\end{array}$} & \multicolumn{8}{|c|}{ Taxa trimestral (\%)/Quarterly rate (\%) } \\
\hline & \multicolumn{4}{|c|}{2014} & \multicolumn{4}{|c|}{2015} \\
\hline & $\begin{array}{l}1{ }^{\circ} \text { tri- } \\
\text { mestre/ } \\
1 s t \\
\text { quarter }\end{array}$ & $\begin{array}{l}2^{\circ} \text { tri- } \\
\text { mestre/ } \\
\text { 2nd } \\
\text { quarter }\end{array}$ & $\begin{array}{l}3^{\circ} \text { tri- } \\
\text { mestre/ } \\
3 r d \\
\text { quarter }\end{array}$ & $\begin{array}{l}4^{\circ} \text { tri- } \\
\text { mestre/ } \\
4 \text { th } \\
\text { quarter }\end{array}$ & $\begin{array}{l}1 \stackrel{0}{ } \text { tri- } \\
\text { mestre/ } \\
1 s t \\
\text { quarter }\end{array}$ & $\begin{array}{l}2{ }^{\circ} \text { tri- } \\
\text { mestre/ } \\
\text { 2nd } \\
\text { quarter }\end{array}$ & $\begin{array}{l}\text { 3o tri- } \\
\text { mestre/ } \\
\text { 3rd } \\
\text { quarter }\end{array}$ & $\begin{array}{l}4{ }^{\circ} \text { tri- } \\
\text { mestre/ } \\
4 \text { th } \\
\text { quarter }\end{array}$ \\
\hline $\begin{array}{l}\text { Produto interno bruto a } \\
\text { preço de mercado / } \\
\text { Gross domestic product at } \\
\text { market prices }\end{array}$ & 3,2 & $(-) 0,8$ & $(-) 1,1$ & $(-) 0,7$ & $(-) 2,0$ & $(-) 3,0$ & $(-) 4,5$ & $(-) 5,9$ \\
\hline $\begin{array}{l}\text { Agropecuária / } \\
\text { Agriculture, forestry and } \\
\text { fishing }\end{array}$ & 6,2 & (-) 0,6 & 0,3 & 2,2 & 5,4 & 2,2 & (-) 2,0 & 0,6 \\
\hline $\begin{array}{l}\text { Indústria / } \\
\text { Manufacturing, mining and } \\
\text { quarrying }\end{array}$ & 4,6 & (-) 2,7 & (-) 2,9 & (-) 2,1 & (-) 4,4 & (-) 5,7 & (-) 6,7 & (-) 8,0 \\
\hline $\begin{array}{l}\text { Serviços / } \\
\text { Services }\end{array}$ & 2,2 & (-) 0,0 & (-) 0,3 & (-) 0,3 & (-) 1,4 & (-) 1,8 & (-) 2,9 & (-) 4,4 \\
\hline $\begin{array}{l}\text { Valor adicionado a preços } \\
\text { básicos / } \\
\text { Value added at basic prices }\end{array}$ & 3,1 & $(-) 0,7$ & $(-) 1,0$ & $(-) 0,7$ & $(-) 1,7$ & $(-) 2,5$ & $(-) 3,8$ & $(-) 5,0$ \\
\hline
\end{tabular}

Fonte/Source: Indicadores IBGE: contas nacionais trimestrais: indicadores de volume e valores correntes: out./dez. 2015. Rio de Janeiro: IBGE, 2016. Disponível em/Available from: < http://www.ibge.gov.br/ home/estatistica/indicadores/pib/defaultcnt.shtm>.Acesso em: mar. 2016/Cited:Mar. 2016.

Notas: 1. Dados preliminares.

2. Variação percentual em relação ao mesmo trimestre do ano anterior./

Notes: 1. Preliminary data.

2. Percent change vis-à-vis the same quarter of previous year. 
Tabela 10.5 - Principais relações macroeconômicas - 2013-2015

Table 10.5 - Main macroeconomic relationships - 2013-2015

\begin{tabular}{lccr}
\hline \multicolumn{1}{c}{$\begin{array}{c}\text { Principais relações/ } \\
\text { Main relationships }\end{array}$} & \multicolumn{2}{c}{ Em percentual (\%)/ Percent (\%) } \\
\cline { 2 - 4 } & 2013 & 2014 & 2015 \\
\hline $\begin{array}{l}\text { Taxa de investimento/ } \\
\text { Investment rate }\end{array}$ & 20,9 & 20,2 & 18,2 \\
Carga tributária bruta/ (1) & 33,6 & - & - \\
Tax burden (1) & & 25,1 & \\
$\begin{array}{l}\text { Grau de abertura da economia/ } \\
\text { Degree of opening of the economy }\end{array}$ & 25,6 & \\
\hline
\end{tabular}

Fonte/Source: IBGE, Diretoria de Pesquisas, Coordenação de Contas Nacionais.

Nota: Os dados de 2014 e 2015 são preliminares baseados em Contas Nacionais Trimestrais./ Note: Preliminary data for 2014 and 2015 based on the Quarterly National Accounts.

(1) Não inclui as contribuições sociais imputadas./ (1) Does not include imputed social. 